\title{
Cardiac Risk Factors for Patients under DSE
}

\section{Rabindra Nath Das}

Department of Statistics, The University of Burdwan, Burdwan, West Bengal, India

*Corresponding author: Rabindra Nath Das, Department of Statistics, The University of Burdwan, Burdwan, West Bengal, India, Tel: +91-9232638970; E-mail: rabin.bwn@gmail.com

Rec date: Aug 17, 2016; Acc Date: September 22, 2016; Pub date: September 28, 2016

Copyright: (c) 2016 Das RN. This is an open-access article distributed under the terms of the Creative Commons Attribution License, which permits unrestricted use, distribution, and reproduction in any medium, provided the original author and source are credited.

\begin{abstract}
This article focuses the cardiac risk factors who underwent Dobutamine stress echocardiography (DSE), based on the data set in UCLA Statistics Web Site. Only the risk factors of different blood pressures, cardiac ejection fractions, and heart rates are discussed for the awareness of the healthy individuals, cardiac patients, and medical practitioners.
\end{abstract}

Keywords: Blood pressure; Cardiac ejection fraction; Coronary heart disease; Gamma model; Heart rate; Non-constant variance

\section{Cardiac Risk Factors}

Hypertension is associated with stroke for $54 \%$, and $47 \%$ of ischaemic heart disease [1], and it affects $30 \%$ of the adult population [2]. An important risk factor of the severity of the systolic heart failure is the ejection fraction (EF) which indicates to the percentage of blood that is pumped (or ejected) out of the ventricles with each heartbeat. A low EF is always associated with the heart disease. Generally, healthy individuals have EF between 50\% and 65\% [3]. Practically, normal values depend on the modality being used to compute the $\mathrm{EF}$, and it may depend on the person's physical, biochemical, lifestyle, working nature, dietary parameters, etc. [4]. Some articles consider the normal values of $\mathrm{EF}$ are between $55 \%$ and $75 \%$ [4,5]. For a normal healthy individual, heart beats 60 to 80 times per minute, while for an athletic or very active, basal heart rate may be lower as the heart pumps more efficiently $[6,7]$.

We seek answers to the following questions: What are the causal factors of different blood pressures, cardiac ejection fractions, and heart rates? What are the association of the risk factors with these cardiac disease markers? These answers are identified based on the data set of 31 variables on 558 subjects (UCLA stress echocardiography data). A detailed description of the data set, collection method, patient population, and the DSE is given in [8]. Effects of dobutamine stress echocardiography have been described in many articles [9-12]. The variables/ factors of this study are Basal heart rate (bpm) (coded as bhr), Basal blood pressure ( $\mathrm{mmHg}$ ) (coded as basebp), Basal double product (DP) (bhr ${ }^{*}$ basebp) (bpm ${ }^{*} \mathrm{mmHg}$ ) (coded as basedp), Peak heart rate (bpm) (coded as pkhr), Systolic blood pressure (mmHg) (coded as sbp), DP pkhr ${ }^{*} \mathrm{sbp}\left(\mathrm{bpm}^{*} \mathrm{mmHg}\right.$ ) (coded as dp), Dobutamine dose (DD) given (coded as dose), Maximum heart rate (bpm) (coded as maxhr), Percent maximum predicted heart rate (coded as pctMphr), Maximum blood pressure ( $\mathrm{mmHg}$ ) (coded as mbp), DP on max DD $\left(\mathrm{bpm}^{*} \mathrm{mmHg}\right.$ ) (coded as dpmaxdo), DD at max double product (mg) (coded as dobdose), Age (years) (coded as age), Gender (male $=0$, female $=1$ ) (coded as gender), Baseline cardiac ejection fraction (coded as baseEF), Ejection fraction on dobutamine (coded as dobEF), Chest pain (yes $(y)=0$, no $(n)=1$ ) (coded as chestpain), Resting wall motion abnormality on echocardiogram (ECDG) $(y=0, n=1)$ (coded as restwma), Positive stress ECDG $(\mathrm{y}=0, \mathrm{n}=1)$ (coded as posSE), New myocardial infraction (MI) $(\mathrm{y}=0, \mathrm{n}=1)$ (coded as newMI), Recent angioplasty $(\mathrm{y}=0, \mathrm{n}=1)$ (coded as newPTCA), Recent bypass surgery $(\mathrm{y}=0, \mathrm{n}=1)$ (coded as newCABG), death $(\mathrm{y}=0, \mathrm{n}=1)$ (coded as death), History of hypertension $(\mathrm{y}=0, \mathrm{n}=1)$ (coded as hxofHT), History of diabetes $(\mathrm{y}=0, \mathrm{n}=1)$ (coded as hxofDM), History of smoking (nonsmoker $=0$, moderate $=1$, heavy $=2$ ) (coded as hxofCig), History of MI $(\mathrm{y}=0, \mathrm{n}=1)$ (coded as hxofMI), History of angioplasty $(\mathrm{y}=0, \mathrm{n}=1)$ (coded as hxofPTCA), History of coronary artery bypass surgery ( $y=0$, $\mathrm{n}=1$ ) (coded as hxofCABG), Death, newMI, newPTCA or newCABG (death $=0$, no=1) (coded as any event), Baseline electrocardiogram diagnosis (normal $=0$, equivocal $=1, \mathrm{MI}=2$ ) ( coded as ecg). This data set has been analyzed by the joint Gamma models [13]. The risk factors of the cardiac markers (blood pressures, cardiac ejection fractions, and heart rates) are as follows.

The mean basal blood pressure (basebp) is separately negatively associated with bhr $(\mathrm{P}<0.01)$, dpmaxdo $(\mathrm{P}<0.01)$, any event $(\mathrm{P}=0.03)$. If bhr or dpmaxdo is low, basebp is high. Basebp is high for the cardiac patients who are close to death. Basebp is separately positively associated with basedp $(\mathrm{P}<0.01)$, maxhr $(\mathrm{P}<0.01)$, mbp $(\mathrm{P}<0.01)$, age $(\mathrm{P}=0.01)$. If at least any one of basedp, maxhr, mbp, age is high, basebp is also high. Mean systolic blood pressure ( $\mathrm{sbp}$ ) is separately negatively associated with bhr $(\mathrm{P}<0.01)$, pkhr $(\mathrm{P}<0.01)$, pctMphr $(\mathrm{P}=0.03)$, dpmaxdo $(\mathrm{P}<0.01)$, hxofHT $(\mathrm{P}=0.05)$. So, sbp is high if bhr or pkhr or pctMphr or dpmaxdo is low. Sbp is higher for the cardiac patients having history of hypertension (hxofHT). Sbp is separately positively associated with basedp $(\mathrm{P}<0.01), \mathrm{dp}(\mathrm{P}<0.01)$, dose $(\mathrm{P}=0.03)$, maxhr $(\mathrm{P}<0.01), \mathrm{mbp}(\mathrm{P}<0.01)$. If any one of basebp, dp, dose, maxhr, mbp is high, sbp is also high. Mean maximum blood pressure (mbp) is separately positively associated with pkhr $(\mathrm{P}<0.01)$, sbp $(\mathrm{P}<0.01)$, dpmaxdo $(\mathrm{P}<0.01)$, age $(\mathrm{P}=0.13)$, posSE $(\mathrm{P}=0.03)$. If at least any one of pkhr, sbp, dpmaxdo, age is high, mbp is also high. The cardiac patients with no positive stress ECDG (posSE) have high mbp. Mean mbp is separately negatively associated with $\mathrm{dp}(\mathrm{P}<0.01)$, maxhr $(\mathrm{P}<0.01)$, newPTCA $(\mathrm{P}=0.02)$, hxofMI $(\mathrm{P}=0.09)$, hxofCABG $(\mathrm{P}=0.05)$. If $\mathrm{dp}$ or maxhr is low, mbp is high. The cardiac patients with newPTCA or hxofMI or hxofCABG have high mbp.

The mean baseline cardiac ejection fraction (baseEF) is separately negatively associated with dp $(\mathrm{P}<0.01)$, dose $(\mathrm{P}=0.02)$, newPTCA $(\mathrm{P}=0.01)$, hxofMI $(\mathrm{P}=0.06)$, ecg $(\mathrm{P}=0.05)$. So, baseEF is low if $\mathrm{dp}$ or 
Citation: Rabindra Nath Das (2016) Cardiac Risk Factors for Patients under DSE. Gen Med (Los Angeles) 4: 270. doi:

Page 2 of 2

dose is high. The cardiac patients with no newPTCA or hxofMI or ecg with MI have low baseEF. The mean baseEF is separately positively associated with pkhr $(\mathrm{P}=0.02)$, sbp $(\mathrm{P}<0.01)$, dobEF $(\mathrm{P}<0.01)$, restwma $(\mathrm{P}<0.01)$, posSE $(\mathrm{P}<0.01)$, hxofPTCA $(\mathrm{P}=0.03)$. If pkhr, sbp, dobEF are low, baseEF is also low. The cardiac patients with restwma or posSE or hxofPTCA have low baseEF. The mean ejection fraction on dobutamine (dobEF) is separately negatively associated with sbp $(\mathrm{P}<0.01)$, posSE $(\mathrm{P}<0.01)$, newMI $(\mathrm{P}<0.01)$, newCABG $(\mathrm{P}<0.01)$, hxofCig $(\mathrm{P}=0.02)$. If sbp is high, dobEF is low. The cardiac patients with heavy smoking status or with no posSE or newMI or newCABG have low dobEF. The mean dobEF is separately positively associated with dp $(\mathrm{P}<0.01)$, dose $(\mathrm{P}=0.01)$, baseEF $(\mathrm{P}<0.01)$, newPTCA $(\mathrm{P}=0.07)$. If $\mathrm{dp}$ or dose or baseEF is low, dobEF is also low. Also the cardiac patients with newPTCA have low dobEF.

The mean basal heart rate (bhr) is separately negatively associated with basebp $(\mathrm{P}<0.01)$, dobdose $(\mathrm{P}=0.074)$, age $(\mathrm{P}=0.003)$, posSE $(\mathrm{P}=0.056)$. If basebp or dobdose or age is high, bhr is low. The cardiac patients with no posSE have low bhr. The mean bhr is separately positively associated with basedp $(\mathrm{P}<0.01)$, pctMphr $(\mathrm{P}<0.01)$, mbp $(\mathrm{P}<0.01)$, dpmaxdo $(\mathrm{P}<0.01)$. If basedp or pctMphr or mbp or dpmaxdo is low, bhr is also low. The mean peak heart rate (pkhr) is separately negatively associated with sbp $(\mathrm{P}<0.01)$ and gender $(\mathrm{P}=0.08)$. The cardiac patients with high sbp or female sex have low pkhr. The mean pkhr is separately positively associated with maxhr $(\mathrm{P}<0.01)$, dpmaxdo $(\mathrm{P}<0.01)$, restwma $(\mathrm{P}=0.04)$, hxofDM $(\mathrm{P}=0.003)$. If maxhr or dpmaxdo is low, pkhr is also low. The cardiac patients with restwma or hxofDM have low pkhr. The mean maximum heart rate (maxhr) is separately positively associated with pkhr $(\mathrm{P}<0.01)$, sbp $(\mathrm{P}<0.01)$, pctMphr $(\mathrm{P}<0.01)$, dpmaxdo $(\mathrm{P}<0.01)$, dobEF $(\mathrm{P}=0.06)$, hxofDM $(\mathrm{P}=0.06)$, hxofPTCA $(\mathrm{P}<0.01)$. If at least any one of pkhr, sbp, pctMphr, dpmaxdo, dobEF is low, maxhr is also low. The cardiac patients with hxofDM or hxofPTCA have low maxhr. The mean maxhr is separately negatively associated with $\mathrm{dp}(\mathrm{P}<0.01), \mathrm{mbp}(\mathrm{P}<0.01)$, and age $(\mathrm{P}<0.01)$. If at least any one of $\mathrm{dp}, \mathrm{mbp}$ and age is high, maxhr is also low.

The above results are derived based on joint Gamma models [13]. Here only the mean parameters of the responses are very shortly discussed. All the derivations along with many mean and dispersion parameters will be discussed in the full research papers. The complete research papers will be submitted very soon. The above mentioned cardiac risk factors are associated with DSE patients. This report recommends the following for all individuals. Care should be taken for blood pressures at older ages. Male individuals have higher hypertension risk than females. Blood pressures and heart rates are highly associated. Smoking should be stopped. Medical practitioners should be care on dobutamine dose, and its interaction effects with other risk factors. For better medical treatment, cardiac events and the history of the disease should be considered by the medical practitioners.

\section{References}

1. Micha R, Wallace SK, Mozaffarian D (2010) Red and processed meat consumption and risk of incident coronary heart disease, stroke, and diabetes mellitus: a systematic review and meta-analysis. Circulation 121: 2271-2283.

2. Kearney PM, Whelton M, Reynolds K, Muntner P, Whelton PK, et al. (2005) Global burden of hypertension: analysis of worldwide data. Lancet 365: 217-223.

3. Kumar V, Abbas AK, Aster J (2009) Robbins and Cotran pathologic basis of disease (8th edn.) St. Louis, Mo: Elsevier Saunders. p. 574.

4. Maceira A (2006) Reference right ventricular systolic and diastolic function normalized to age, gender and body surface area from steadystate free precession cardiovascular magnetic resonance. Eur Heart J 27: 2879-2888.

5. Maceira A (2006) Normalized left ventricular systolic and diastolic function by steady state free precession cardiovascular magnetic resonance. J CARDIOV MAGN RESON 8: 417-426.

6. Cole CR, Foody JM, Blackstone EH, Lauer MS (2000) Heart rate recovery after submaximal exercise testing as a predictor of mortality in a cardiovascularly healthy cohort. Ann Intern Med 132: 552-555.

7. Whyte GP, George K, Nevill A, Shave R, Sharma S, et al. (2004) Left ventricular morphology and function athletes: a meta-analysis. Int J Sports Med 25: 380-383.

8. Krivokapich Janine, Child Jhon S, Walter Donald O, Garfinkel Alan (1999) Prognostic value of dobutamine stress echocardiography in predicting cardiac events in patients with known or suspected coronary artery disease. J Am Coll Cardiol 33: 708-716.

9. O'Driscoll JM, Rossato C, Gargallo-Fernandez P (2015) The prognostic value of dobutamine stress echocardiography amongst British Indian Asian and Afro-Caribbean patients: a comparison with European white patients. Cardiovasc Ultrasound 13: 36-41.

10. Aboukhoudir F, Rekik S, Latil Plat F (2014) Uselessness of high-sensitivity cardiac troponins to improve diagnostic accuracy of dobutamine stress echocardiography in high-risk diabetic patients. Ann Cardiol Angeiol 63: 307-311.

11. Yao SS, Supariwala A, Yao A (2015) Prognostic value of stress echocardiography in patients with low-intermediate or high short-term (10 years) versus low (<39\%) or high ((yen)39\%) lifetime predicted risk of cardiovascular disease according to the American College of Cardiology/ American Heart Association 2013 cardiovascular risk calculator. Am J Cardiol 116: 725-729.

12. van der Sijde JN, Boiten HJ, van Domburg RT (2016) Long-term (> 10 Years) prognostic value of dobutamine stress echocardiography in a highrisk cohort. Am J Cardiol 117: 1078-1083.

13. Das RN (2014) Robust response surfaces, regression, and positive data analyses. CRC Press: Chapman \& Hall, London. 